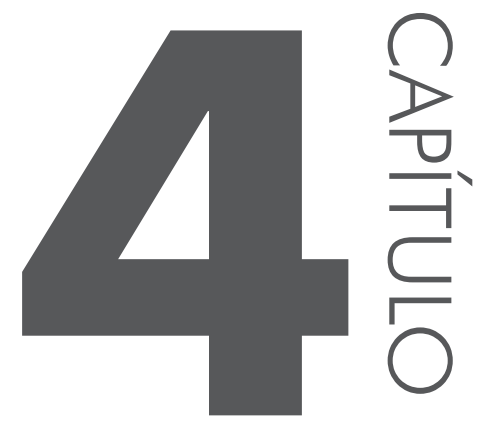

\title{
BARREIRAS PARA IMPLANTAÇÃO E MANUTENÇÃO DAS PRÁTICAS DE GREEN SUPPLY CHAIN MANAGEMENT
}

Bianca Dias da Silva ${ }^{1}$ Maico Roris Severino ${ }^{1}$

Agradecimentos: Ao Conselho Nacional de Desenvolvimento Científico e Tecnológico (CNPq) pela concessão da bolsa de estudos do Programa Institucional de Bolsa Voluntária de Iniciação Científica (PIVIC).

1 Unidade Acadêmica Especial de Gestão e Negócios, Universidade Federal de Goiás - Regional Catalão, Catalão, Brasil. E-mail de contato: bianca.dias.silvaa@gmail.com, maicororis@gmail.com 
Resumo: A sustentabilidade tornou-se um tema de grande relevância no contexto mundial para esta e as futuras décadas. Em decorrência deste fato, de uma maneira geral as organizações se apresentam mais conscientes e interessadas em adotar práticas ambientais visando obter vantagem competitiva. Dentre as práticas, destaca-se a Green Supply Chain Management (GSCM), ou Gestão da Cadeia de Suprimentos Verde. Contudo, verifica-se que a implementação destas ações na indústria ainda é tímida. Assim, este estudo objetivou analisar as principais barreiras com as quais as organizações deparam ao optarem por implementar práticas relacionadas à GSCM, bem como efetuar a sua manutenção, uma vez que a implementação foi bem-sucedida. Dentre as principais barreiras identificadas, pode-se destacar legislação, custo, questões relacionadas a clientes/ consumidores, entre outros. O estudo apresentou uma diversidade de desafios de distintas naturezas, sobretudo para que a implementação seja efetiva, demonstrando a relevância de estudos e a busca por alternativas para solucionar ou amenizar as consequências impostas estas barreiras.

Palavras-chave: Green Supply Chain Management. Barreiras. Implementação. Manutenção.

Abstract: Sustainability has become a relevant subject in a worldwide context for this and the next future decades. Due to this fact in a general way the organizations show themselves aware and more interested in adopting environmental practices aiming competitive advantage. Among this practices, we can highlight the Green Supply Chain Management (GSCM). However, the implementation of these practices by the industries is still irrelevant. This way, this study aims to analyze the main barriers that organizations face when they opt for implementing practices related to GSCM and make the maintenance of these practices, once this implementation is successful. The study shows a diversity of challenges of distinct nature, so that the implementation is effective, demonstrating the relevance of studies and the search for alternatives to solve or mitigate the consequences imposed to these barriers.

Keywords: Green Supply Chain Management. Barriers. Implementation. Maintenance.

\section{INTRODUC̣ÃO}

A sustentabilidade tem se tornado um fator decisivo para competitividade nas últimas décadas, assim como se presume que também seja uma tendência futura. Desta forma, torna-se vital para a sobrevivência das organizações a implementação de práticas que reduzam os impactos relativos às atividades industriais. Associada a medidas legislativas rigorosas e crescente consciência ambiental em um âmbito mundial, é possível observar uma intensa pressão exercida sobre as 
organizações, o que torna imprescindível formular estratégias que melhorem o desempenho ambiental das empresas (ZHU; SARKIS; GENG, 2006).

Desta maneira, verifica-se que é fundamental considerar o tema sustentabilidade e meio ambiente como parte da cultura das organizações, buscando a implementação de práticas "verdes" por toda a cadeia de suprimentos. Seuring e Müller (2011) destacam que os integrantes de uma a cadeia de suprimentos estão irrevogavelmente conectados por meio dos fluxos de materiais, de informação e de capital, fazendo com que os componentes de toda a cadeia de suprimentos sejam responsáveis por implantar e manter as práticas sustentáveis, não sendo responsabilidade apenas das empresas focais.

A Green Supply Chain Management (GSCM), ou Gestão da Cadeia de Suprimentos Verde, pode ser definida como uma área interdisciplinar que nos últimos anos se desenvolveu extensamente, ganhando notoriedade tanto na indústria quanto no meio acadêmico (SARKIS, 2005). Deste modo, a mudança na estratégia da cadeia de suprimentos foi de suma importância para que houvesse a ascensão da GSCM, fazendo com que esta pudesse se desenvolver como um campo crescente e separado, propondo uma integração ambiental de conceitos (MUDULI apud SHRIVASTAVA, 2013).

Portanto, a GSCM emerge como uma ferramenta de gerenciamento altamente eficiente e também como uma espécie de filosofia direcionada às organizações que lideram a implementação destes conceitos por toda a cadeia, almejando alcançar desde a compra "verde", integrada ao gerenciamento do ciclo de vida do produto para que envolva desde o fornecedor até o fabricante e o cliente, fechando o ciclo com a logística reversa (ZHU; SARKIS, 2004). Segundo Gilbert (2000), a GSCM aborda o conceito da cadeia de suprimentos tradicional integrada a fundamentos ambientais, visando manter uma relação em longo prazo com os fornecedores e implementar a logística reversa, bem como tomar decisões conjuntas de compras ambientais. Esta prática busca limitar os desperdícios referentes aos processos industriais de forma a conservar energia e outros recursos, além de evitar que haja dispersão de resíduos perigosos no meio ambiente (NUNES; MARQUES; TORRES, 2004). Cada vez mais este conceito vem sendo incorporado pela indústria, tornando-se uma forma das organizações trabalharem de acordo com as exigências dos regulamentos ambientais para melhorar o desempenho dos processos produtivos e de produtos (HSU; HU, 2008) para reduzir os impactos ambientais inerentes às atividades industriais. Existem diversos motivos que podem levar uma organização a adotar a GSCM, pois, além da redução de impactos ambientais, pode haver também um retorno financeiro positivo para a organização que adote estas práticas (HALL, 2000).

Apesar de haverem benefícios econômicos e sociais a serem obtidos por meio da adoção e implementação da GSCM na indústria, grande parte das organiza- 
ções demonstra relutância em adotar estas práticas. Esta situação se deve ao fato de que, durante a implementação da GSCM, as empresas se deparam com diversos desafios e problemas para conseguir, efetivamente, atingir o resultado almejado (BARVE et al., 2013). Barreiras podem ser definidas de maneira geral, como aqueles fatores que impedem e interferem na adoção eficaz de práticas ambientais nas organizações e em suas respectivas cadeias de suprimentos (PERRON, 2005). Alguns destes obstáculos podem ser observados e prevenidos de maneira mais eficaz durante o estágio inicial da implantação da GSCM, desde que as empresas se mostrem preparadas para erradicar estas barreiras, reconhecendo que nem sempre é possível remove-las de forma simultânea (GOVINDAN; KALIYAN; KANNAN, 2013).

Em vista disso, é de grande relevância identificar as barreiras encontradas durante a implantação de forma a remover estes problemas (SARKIS; ZHU, 2008) e para realizar a manutenção de práticas sustentáveis por toda a cadeia de suprimentos. A implementação efetiva na indústria desempenha um importante papel no quesito vantagem competitiva, tanto em ganhar quanto em mantê-la. Neste sentido, o objetivo deste estudo foi de realizar uma análise das barreiras e desafios com que as organizações de diversos segmentos da indústria mundial se deparam ao optar pela implementação de práticas ambientais relacionadas à GSCM.

\section{METODOLOGIA}

O tipo de pesquisa utilizada neste estudo foi a teórico-conceitual, sendo a parte teórica realizada por meio de pesquisa bibliográfica. Para a condução do estudo bibliográfico, foram consultadas diversas publicações que abordam o tema Green Supply Chain Management. A finalidade deste levantamento bibliográfico é permitir maior conhecimento ao pesquisador acerca das diversas barreiras encontradas pelas organizações de múltiplos segmentos da indústria mundial, cada qual com seu desafio, e, ainda, conhecer quais práticas que estão sendo adotadas pelas empresas para solucionar estes problemas.

A pesquisa foi conduzida no período de 01/02/2015 a 05/02/2015, e os artigos foram selecionados por meio da base de dados do website Engineering Village, utilizando-se da combinação do termo "Green Supply Chain Management" com as palavras "maintenance", "implemente", "barriers" e "challenges", encontrando aproximadamente 722 artigos, dentre os quais foram selecionados 61 . Estas publicações foram escolhidas por melhor corresponderem aos objetivos da pesquisa, ou seja, os artigos selecionados abordam de maneira adequada e conveniente as barreiras e os desafios encontrados pelas organizações para a implementação da GSCM.

A partir da seleção dos artigos, foi realizada a análise das publicações com ênfase nos estudos de caso. Portanto, após a realização da leitura, foi elaborada 
uma lista, com o objetivo de organizar a coleta e facilitar a análise dos dados, extraindo os principais motivadores que levaram as organizações a implementarem práticas da GSCM, as principais barreiras e os desafios encontrados ao longo do período de implantação e manutenção, como também as práticas adotadas para solucioná-los. Por meio da utilização dos dados extraídos dos artigos, foi possível enumerar a frequência com que as barreiras se apresentam e posteriormente, realizar uma análise gráfica, demonstrando quais são as barreiras mais comuns encontradas pelas organizações durante diversos estágios da implantação de práticas sustentáveis em toda a cadeia de suprimentos e posteriormente, sugestões para solucioná-las ou minimizá-las.

\section{RESULTADOS}

Após análise minuciosa de publicações selecionadas, pôde-se listar ao todo 29 barreiras. Aquelas analisadas e discutidas neste estudo possibilitam uma visão das principais barreiras encontradas no processo de implantação e manutenção da GSCM. No decorrer da análise, observou-se a repetição das mesmas barreiras em vários artigos científicos, o que tornou possível a sistematização de dados e a construção de um gráfico. Este, por sua vez, tem o intuito de facilitar a visualização e, por conseguinte, a compreensão dos dados obtidos por meio da revisão da literatura, como se pode verificar na Figura 1.

Como apresentado na Figura 1, é possível observar quais barreiras foram relatadas na literatura com maior frequência. Neste contexto, foram escolhidas para análise as barreiras que se repetiam em mais de nove artigos, por considerá-las com maior relevância em função de suas recorrências. São elas: legislação, custos, questões relacionadas a clientes e consumidores, questões relacionadas a fornecedores, capacidade produtiva e estratégia adotada pela organização. Cada barreira é detalhada a seguir.

A barreira legislação foi a que se apresentou com maior frequência dentre os estudos de caso observados. Como a capacidade de estabelecer regulamentos ambientais para a indústria está centrada no governo, pode-se afirmar que este pode incentivar ou não a indústria como um todo a adotar práticas ambientais (SCUPOLA, 2003). A maior parte das empresas, principalmente as microempresas, encaram a questão ambiental como um custo, isto é, um fator que influencia diretamente na lucratividade da empresa (PETTS et al., 1999). Outro aspecto relatado pelas empresas é a necessidade de uma alta demanda de produtos considerados "verdes", o que consequentemente pode aumentar o custo final de venda. As constantes mudanças na legislação ambiental são um tópico recorrente, pois há dificuldade em interpretar e manter conformidade com as leis ambientais (BAYLIS, CONNELL, FLYNN, 1998), bem como falta de incentivo do governo, 
que comumente recomenda práticas obsoletas e, diversas vezes, ineficazes (AL KHIDIR; ZAILANI, 2009). Também se devem evidenciar taxas, tempo utilizado para que a empresa esteja sempre em conformidade com as normas e altos impostos (LUTHRA et al., 2011). Assim sendo, a legislação se apresenta como uma barreira pois influencia diretamente na competitividade e na lucratividade de uma organização e, consequentemente, em toda a cadeia de suprimentos.

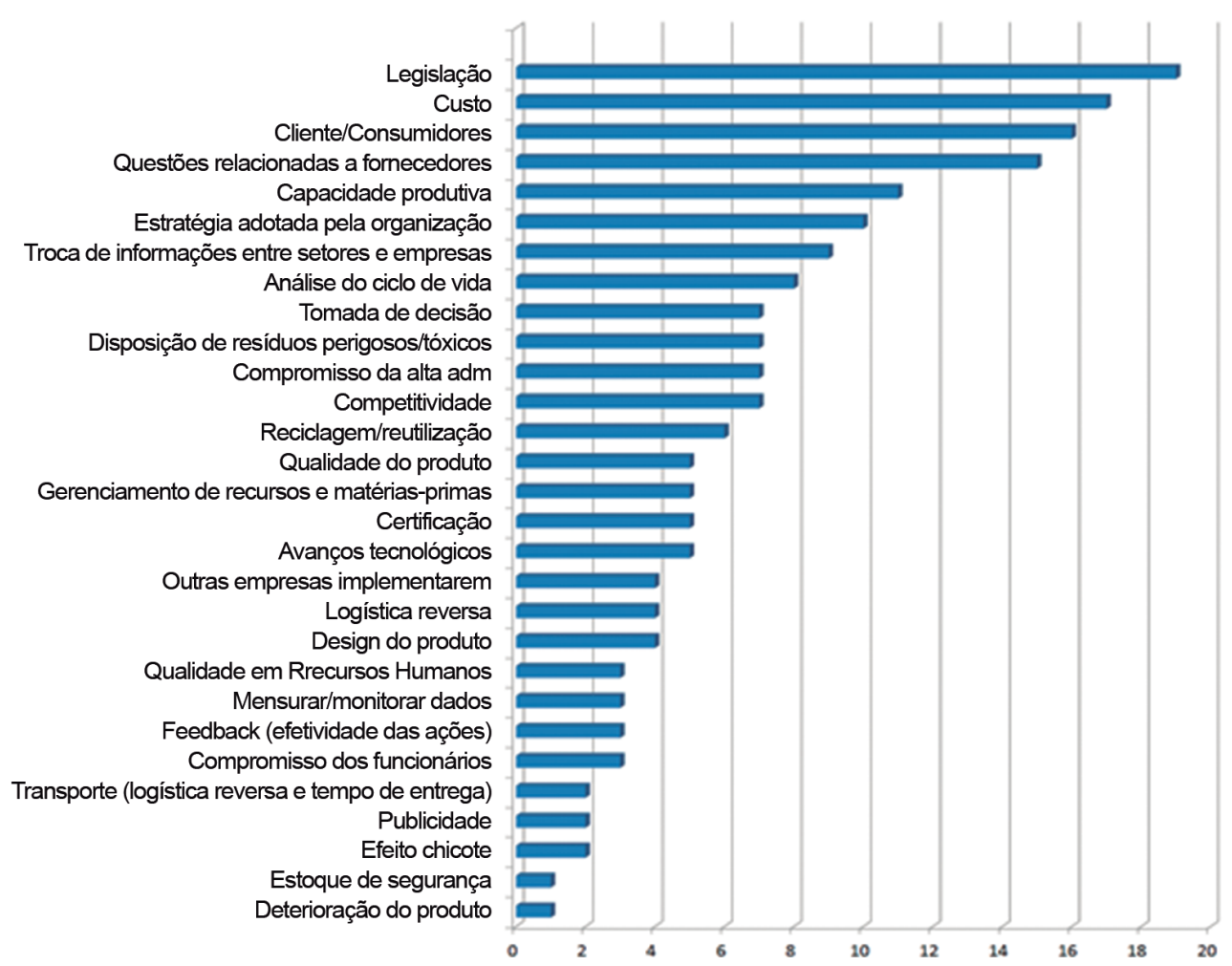

Figura 1. Análise da frequência com que as barreiras listadas foram relatadas nos casos analisados. 0 gráfico foi estruturado para mostrar na ordem decrescente a repetição das barreiras por número de artigos.

De um modo geral, o custo é também uma barreira crucial para o desenvolvimento de práticas ambientais nas empresas. É possível observar que todas as outras barreiras estão relacionadas com o custo. As organizações de todo o mundo utilizam este fator como uma maneira de mensurar sua performance de forma que o custo na GSCM seja um aspecto de grande pressão se comparado à cadeia de suprimentos tradicional (LUTHRA et al., 2011). Estas empresas que planejam e implementam, ou mesmo aquelas que estão buscando implementar, a GSCM devem, simultaneamente, conseguir a redução de custos e dos impactos ambientais inerentes às atividades industriais, bem como aumentar o lucro e a eficiência da 
cadeia de suprimentos e, ainda, o market share (VAN HOEK, 2000). Conforme mencionado, as alterações a serem realizadas para que a cadeia de suprimentos se adeque aos objetivos sustentáveis podem impactar nos custos de produção e, consequentemente, no preço final deste produto. Em outras palavras, o custo de tornar o produto ambientalmente "correto" será repassado para o cliente em virtude das alterações realizadas na produção. $\mathrm{O}$ investimento inicial a ser feito é excessivamente alto, o que faz com que as companhias se considerem menos competitivas em relação às concorrentes que não adotam práticas ambientais em curto prazo. Ademais, é relatado na literatura que, a longo prazo, a implantação de práticas de GSCM se demonstram economicamente atrativas.

As questões relacionadas aos clientes/consumidores apresentam-se como a terceira barreira de maior ocorrência. É necessário ressaltar que o consumidor é a base e a essência de qualquer mercado, assim, as corporações buscam de todas as formas atender às suas necessidades e satisfazê-los de qualquer maneira. Apesar da consciência ambiental por parte da sociedade apresentar um crescimento considerável nas últimas décadas, o maior obstáculo para as empresas se tratando dos clientes é o fato de não haver uma grande demanda por produtos "verdes" (MUDULI, 2014). O número de consumidores conscientes ainda não é suficientemente significativo, pois diversas vezes o consumidor tem uma compreensão equivocada por não ter conhecimento do termo, considerando se tratar de opção de cor da empresa ou até mesmo com foco em vegetarianos (WANG et al., 2013). Assim, este fator se apresenta também como uma forte barreira, pois o custo em tornar um produto "verde" é alto, resultando na alteração de preço destes produtos, que se torna mais elevado do que o da concorrência. Por não haver a demanda necessária que opte em consumir este produto, por conta do preço elevado em relação ao produto tradicional, as empresas perdem para a concorrência. A falta de publicidade e parcerias entre as organizações e os clientes também são fatores que contribuem para que os consumidores se tornem uma barreira (JABBOUR et al., 2014).

Para o efetivo funcionamento de uma cadeia de suprimentos, cada empresa deve ser responsável pelo desempenho tanto social quanto ambiental de seus fornecedores (SEURING; MULLER, 2008). Portanto, as questões relacionadas aos fornecedores assumem grande relevância para que se incentive a adoção de práticas "verdes" em todos os níveis de uma cadeia de suprimentos em decorrência do planejamento ambiental a ser feito, firmando-se uma parceira a longo prazo para compartilhar o conhecimento com os outros componentes da cadeia com objetivo final de obter um desempenho financeiro positivo de suas práticas (VACHON; KLASSEN, 2007). Portanto, visa-se facilitar a coordenação das atividades, como também mudar os processos de maneira a ganhar em competitividade e satisfazer os clientes. As questões relacionadas aos fornecedores apresentam-se como uma 
barreira na indústria, pois grande parte deles se recusa a formar uma parceria com o intuito de aderir às iniciativas ambientais, que geralmente são propostas pelas empresas focais. Também há os casos em que os fornecedores concordam com as práticas, no entanto, deixam de implementá-las de fato. Outro relato é de casos relativos à tecnologia em que, mesmo em uma cadeia de suprimentos tradicional, o próprio fornecedor tem receio de passar informações para as outras empresas da cadeia, o que dificulta a relação da parceria (GOVINDAN et al., 2014 apud. CALLEJA et al., 2004), bem como a manutenção dos fornecedores "verdes" realizando medições e monitoramentos (GOVINDAN; KALIYAN; KANNAN, 2014). Essa relutância em aderir às práticas da GSCM, mais uma vez, surge em função do custo, do tempo e da disposição para alterar o processo e o produto.

As organizações que se dispõem a serem pioneiras na inovação de produto e processo têm a oportunidade de reforçar sua liderança no mercado, isto é, investimentos que podem levá-las à frente da concorrência em virtude da iniciativa, da qualidade e da imagem pública (VACHON; KLASSEN, 2007). Contudo, o investimento financeiro para novas tecnologias, disposição de tempo para adequação, treinamentos e a habilidade de atrair profissionais que possuem capacidade e conhecimento (O'CONNOR; TURNHAM, 1991) tornam a capacidade produtiva uma barreira complexa a ser solucionada. Ressaltando o objetivo principal das organizações como o lucro, pode-se destacar os obstáculos relacionados ao pouco investimento financeiro para equipamentos e outras aplicações tornam a capacidade produtiva das organizações muito restrita, além de reduzir o lead time (KANNAN et al., 2013). Também, os poucos níveis de economia de escala disponíveis para a produção relativos aos produtos "verdes" acarretam um aumento do preço, o que reduz o output por unidade, além de aumentar o desperdício de recursos (DAS, 2009). Portanto a capacidade produtiva restringe a produção, reduz a flexibilidade e, consequentemente, não atinge seu objetivo primordial: a satisfação dos clientes.

A consciência ambiental dos clientes, como já afirmado, vem aumentando gradativamente, fazendo com que as organizações que implementam práticas da GSCM tenham uma melhor imagem no mercado. Aos poucos, nota-se que as empresas reconhecem a gestão ambiental como uma estratégia-chave para um bom desempenho organizacional (BARVE et al., 2013). O planejamento estratégico fornece um modelo que facilita a tomada de decisões do gestor, além de auxiliar na avaliação da performance organizacional e destacar os erros ou riscos mais significantes para implementação que possam surgir (MUDGAL et al., 2010). Em outras palavras, o desempenho mensurado indica se a companhia deveria continuar ou não a estratégia adotada, fazer ajustes ou mudá-la completamente (BOND, 1999). Diversas organizações que optam pelas práticas ambientais em sua cadeia de suprimentos fazem seu planejamento estratégico com a abordagem 
errada, podendo-se destacar a tomada de decisão em relação ao grau de mudanças a serem realizadas. Aspectos como pouco investimento na motivação, compreensão e capacitação do corpo de funcionários (OLUGU et al., 2010), falta de comprometimento da alta gerência, que ignora os objetivos e as ações ambientais, e reavaliação das práticas antes da implementação de fato contribuem para que uma estratégia seja malsucedida (BARVE et al., 2013). Portanto, a abordagem errônea utilizada pela organização perante o mercado pode acarretar severos prejuízos para a empresa, tornando-se assim uma barreira na implantação da GSCM.

Além das barreiras analisadas anteriormente, podem-se destacar também outras barreiras de grande relevância. Dentre elas a certificação ambiental, que se apresenta como uma barreira pela dificuldade em manter conformidade com as normas, além de seus custos. Também se pode ressaltar a barreira feedback das ações ambientais implementadas, em que algumas vezes se torna complexo notar o diferencial obtido por meio da implementação de maneira nítida, desestimulando a adoção da GSCM. O compromisso da alta administração, que está relacionado diretamente com a barreira anterior, na qual a alta administração não toma medidas para solução das barreiras ou não observa os benefícios das práticas ambientais, reduzindo ou eliminando os investimentos para a manutenção das práticas ambientais. Outras empresas implementarem também é uma barreira comumente relatada, de forma que as organizações não consideram justo competirem com as empresas de produtos tradicionais, que aperfeiçoaram seu modelo de produção, além de não terem gastos com investimentos e manutenção de produtos "verdes". A tomada de decisão é outra barreira de grande impacto na organização, de forma que o gestor não consegue tomar uma decisão eficaz, acarretando em outros problemas e visíveis prejuízos.

Quanto ao design do produto, pode-se evidenciar a falta de flexibilidade das empresas em realizar alterações para se adequarem às práticas ambientais e facilitarem a logística reversa. A barreira avanços tecnológicos está diretamente relacionada com a capacidade produtiva, em que a grande dificuldade está no acompanhamento das inovações em maquinários e equipamentos pelo custo do investimento inicial a ser realizado. A barreira qualidade do produto se destaca pela dificuldade em manter um padrão de qualidade em toda a cadeia de suprimentos, principalmente pelas mudanças a serem efetuadas para obter conformidade ambiental. A disposição de resíduos tóxicos ou perigosos expõe a dificuldade em alterar a produção para se adequar a uma nova matéria-prima, o que pode elevar o preço do produto final ou mesmo acarretar defeitos.

O estoque de segurança é uma barreira também pelo custo, isto é, o custo de manter um estoque de segurança já é alto e, como é comum a mudança no produto para obter economias de escala, acarretam-se custos de obsolescência. A reutilização/reciclagem se apresenta como uma barreira por grande parte das 
empresas focais terem dificuldade em confiar informações e revenda ao provedor logístico por receio de que este último revenda o produto com defeitos ou erros, fazendo com que a empresa perca clientes por conta dos erros do provedor.

Ressalta-se que podem existir outras barreiras relacionadas à implantação e à manutenção da GSCM, além das relatadas anteriormente. Contudo, este estudo se restringiu à análise das barreiras encontradas nos 61 casos, acreditando-se que estas sejam as principais.

\section{CONCLUSÃO}

O objetivo do estudo foi realizar uma análise das barreiras e dos desafios com que as organizações de diversos segmentos da indústria mundial deparam ao optar pela implementação de práticas ambientais relacionadas à GSCM. A partir da realização do estudo, pode-se observar que, em função da complexidade das práticas da GSCM, implementar este conceito torna-se uma tarefa árdua para as organizações e seus respectivos gestores. Ao optar pela adoção destas mesmas práticas, verifica-se o surgimento de diversas barreiras, isto é, obstáculos que impossibilitam um funcionamento eficaz da GSCM. Estes desafios se apresentam frequentemente nos estágios iniciais da implantação na cadeia de suprimentos; contudo, observa-se também que podem surgir posteriormente, na manutenção destes conceitos. Assim, essas barreiras fazem com que as organizações não tenham uma boa visão do potencial competitivo que optar por aderir à GSCM pode acarretar. As empresas estão sendo cada vez mais pressionadas, tanto pelo governo quanto pela sociedade, para implementar práticas sustentáveis por todo o seu processo produtivo. Assim, observa-se a importância de estudos relacionados à remoção ou à minimização dos efeitos negativos ocasionados por estas barreiras intrínsecas à implementação da GSCM, para que as empresas insistam nestas medidas e para encorajar outras empresas a adotarem-nas também.

A barreira que se apresentou com mais frequência, portanto a de maior relevância, é a legislação. Faz-se necessária a proposição de alterações nas legislações e na forma como são aplicadas. É relatado por grande parte das organizações que as legislações ambientais são consideradas fracas e voláteis. As empresas que realmente se empenham em implantar práticas sustentáveis vão muito além do estabelecido, o que pode fazer com que o preço do produto aumente de forma significativa e, portanto, perca em competitividade. Destaca-se a importância de o governo oferecer benefícios para aquelas organizações que decidam implantar práticas sustentáveis, propondo vantagens para que estas empresas se tornem mais competitivas, com ações como a isenção de impostos de forma que o valor a ser arrecadado seja diluído nos custos, e, consequentemente, incentivando outras organizações a adotarem-nas também. Outro fator-chave seria estabelecer leis 
ambientais "padronizadas" e com poucas alterações, de forma que as empresas que exportam produtos consigam se manter em conformidade com as exigências de outros países.

Diversas vezes, é observado que o próprio consumidor não possui consciência sobre o que é um produto "verde" ou o que significam aquelas certificações e, pela diferença de preço, optam pelo produto tradicional da concorrência. Neste contexto, é de imensa importância o desenvolvimento de campanhas publicitárias com o objetivo de difundir o conceito "verde" entre toda a população, tanto por ações governamentais quanto das próprias companhias. Portanto, é necessário um trabalho de conscientização com o cliente sobre a diferença do tradicional e a importância de existir uma demanda para este tipo de alternativa, de forma que deixem de considerar apenas o preço e visem a preservação de recursos naturais. Consequentemente, isso desencadeará uma melhora na competitividade da organização, criando assim a "demanda verde" necessária para a continuação das atividades da organização, assim como estímulo para outras empresas também aderirem a estas práticas.

A necessidade de trabalhar em conjunto almejando o mesmo objetivo, seja eficiência operacional ou redução de desperdícios, buscando criar uma parceria de longo prazo, deve ser a premissa para as empresas focais e seus fornecedores, pois, apesar das várias vantagens, os fornecedores não estão dispostos a mudar a produção em decorrência dos quesitos ambientais. A primeira etapa a ser determinada é a alta qualidade em toda a cadeia de suprimentos. Da mesma forma, estabelecer uma relação de confiança, compartilhar as informações necessárias ao fornecedor para reduzir e eliminar os impactos ambientais, além de investimentos em capacitação e conscientização de funcionários e gestores são medidas que promovem um funcionamento dinâmico dos componentes da cadeia.

A capacidade produtiva está diretamente associada ao desenvolvimento tecnológico. A empresa que escolhe não efetuar os investimentos necessários em novas tecnologias e, assim, aumentar sua economia de escala limita sua capacidade produtiva drasticamente. Reduzir a capacidade produtiva implica na redução do lead time do produto e permite que a concorrência se sobressaia. Portanto, as organizações devem buscar lucratividade a longo prazo e promover a imagem organizacional por meio da inovação tecnológica. Investimentos que mudem o maquinário, reduzam o desperdício, aumentando a flexibilidade e provando que as empresas que investem em sustentabilidade estão aptas a permanecer no mercado.

O planejamento estratégico, por sua vez, deve ser realizado com cautela. É necessário realizar um estudo minucioso e uma simulação do impacto recorrente à sua implantação. Uma estratégia bem definida e estruturada pode destacar aspectos negativos e erros na implementação efetiva da GSCM, bem como facilitar a decisão do gestor nos quesitos ambientais. Destaca-se também a obtenção do total apoio 
da alta administração para que a estratégia projetada seja bem-sucedida e para que haja investimentos futuros para a sua continuação. É importante ressaltar que a estratégia organizacional deve abranger os funcionários da empresa, que são parte vital para que a estratégia seja eficiente e apresente os resultados esperados.

Dessa maneira, observa-se que a tomada de decisão pela solução ou minimização se torna uma reação em cadeia. Portanto, é possível observar que as possíveis soluções estão interligadas e ao adotar uma medida para solucionar um determinado obstáculo, simultaneamente soluciona-se outro. Em consequência, as organizações devem superar os desafios impostos para a manutenção de práticas da GSCM, desde que sejam competitivas e sustentáveis.

Ressalta-se que a presente pesquisa foi estritamente literária, portanto, para estudos futuros, é sugerida a realização de estudos de caso, abordando vários segmentos industriais, com o objetivo de observar a implementação das propostas estabelecidas nesta análise, verificando na prática a validade e o impacto das sugestões na cadeia de suprimentos. Pode-se perceber também que duas barreiras se destacaram, a legislação e o custo, e, futuramente, trabalhos podem ser desenvolvidos com ênfase no impacto desses fatores em uma cadeia de suprimentos real.

\section{REFERÊNCIAS}

AL KHIDIR, T.; ZAILANI, S. Going green in supply chain towards environmental sustainability. Global Journal of Environmental Research, v. 3, n. 3, p. 246-251, 2009. BARVE, A.; MUDULI, K. Modelling the challenges of green supply chain management practices in Indian mining industries. Journal of Manufacturing Technology Management, v. 24, p. 1102-1122, 2013.

BAYLIS, R.; CONNELL, L.; FLYNN, A. Company size, environmental regulation and ecological modernization: further analysis at the level of the firm. Business Strategy and the Environment Bus. Strat. Env., v. 7, p. 285-296, 1998.

BOND, T. The role of performance measurement in continuous improvement.

International Journal of Operations and Production Management, v. 19, n. 12, p. 13181334, 1999.

CALLEJA, I.; DELGADO, L.; EDER, P.; KROLL, A.; LINDBLOM, J.; WUNNIK, C. V.; WOLF, O., GOUARDERES, F.; LANGENDORFF, J. Promoting environmental technologies: sectoral analyses, barriers and measures. IPTS Report EUR 21002EN. Institute for Prospective Technological Studies, Sevilha, 2004.

DAS, A. Does firm ownership differentiate environmental compliance: Evidence from Indian chromite mining industry. Center for Development Studies, Thiruvananthapuram, India, ago. 2009.

GILBERT, S. Greening Supply Chain: Enhancing competitiveness through green productivity. Top Forum on Enhancing Competitiveness through Green Productivity, 2000. 
GOVINDAN, K.; KALIYAN, M.; KANNAN, D. Barriers analysis for green supply chain management implementation in Indian industries using analytic hierarchy process. International Journal Production Economics, v. 147, p. 555-568, 2014.

JABBOUR, A. B.; JABBOUR, C.; GOVINDAN, K.; KANNAN, D.; ARANTAES, A. F. Mixed methodology to analyze the relationship between maturity of environmental management and the adoption of green supply chain management in Brazil. Resources, Conservation and Recycling, v. 92, p.255-267, 2014.

HALL, J. Environmental supply chain dynamics. Journal of Cleaner Production, v. 8, n. 6, p. 455-471, 2000.

HSU, C. W.; HU, A. H. Green Supply Chain Management in the electronic industry. International Journal of Science and Technology, v. 5, n.2, p. 205-216, 2008.

KANNAN, D.; JABBOUR, A. B. L. S.; JABBOUR, C. J. C. Selecting green suppliers based on GSCM practices: Using fuzzy TOPSIS applied to a Brazilian electronics company. European Journal of Operational Research, v. 233, p.432-447, 2013.

LUTHRA S.; KUMAR V.; KUMAR S.; HALEEM A. Barriers to implement green supply chain management in automobile industry using interpretive structural modeling technique - An Indian perspective. Journal of Industrial Engineering and Management, v. 4, n. 2, 2011.

MUDGAL, R. K.; SHANKAR, R.; TALIB, P.; RAJ, T. Modeling the barriers of green supply chain practices: an Indian perspective. Int. Journal of Logistics Systems and Management, v. 7, n. 1, p. 81-107, 2010.

NUNES, B. T. S.; MARQUES, S.; RAMOS, R. E. B. A theoretical approach for Green Supply Chain. Universidade Federal do Rio Grande do Norte, 2004.

O'CONNOR, D.; TURNHAM, D. Environmental management in developing countries: an overview. Development and International Co-operation, v. 3, n.13, p.75-100, 1991.

OLUGU, E. U.; WONG, K. Y.; SHAHAROUN, A. M. Development of key performance measures for the automobile green supply chain. Resources Conservation and Recycling, v. 55, p. 567-579, 2010.

PERRON, G. M. Barriers to environmental performance improvements in Canadian SMEs. Dalhousie University, Canada, 2005

PETTS, J.; HERD, A.; GERRARD, S.; HORNE, C. The climate and culture of environmental compliance within SMEs. Business Strategy and the Environment, v. 8, n.1, p. 14-30, 1999.

SARKIS, J. A boundaries and flows perspective of Green Supply Chain Management. Supply Chain Management: An International Journal, v. 17, n. 2, p. 202216, 2011.

SARKIS, J.; ZHU Q. An inter-sectoral comparison of green supply chain management in China: Drivers and practices. Journal of Cleaner Production, v. 14, p. 472-486, 2005.

SARKIS, J.; ZHU Q.; LAI, K. H. Confirmation of a measurement model for green supply chain management practices of implementation. International Journal of Production Economics, v. 111, n. 2, p. 261-273, 2008. 
SEURING, S.; MULLER, M. From a literature review to a conceptual framework for sustainable supply chain management. Journal of Cleaner Production, v. 16, p. 16991710, 2001.

SCUPOLA, A. The adoption of internet commerce by SMEs in the South of Italy: an environmental, technological and organizational perspective. Journal of Global Information Technology Management, v. 6, n. 1, p. 52-71, 2003.

SHRIVASTAVA, P. Greening business: profiting the corporation and the environment. Cincinnati: Thompson Executive Press, 1995.

VACHON, S.; KLASSEN, R. Green project partnership in the supply chain: the case of the package printing industry. Journal of Cleaner Production, v. 14, p. 661-671, 2005.

Supply Chain Management and environmental technologies: the role of integration. International Journal of Production Reasearch, v. 45, p.401-423, 2007. VAN HOEK, R. From reversed logistics to green supply chains. Logistic Solutions, v. 4, p. 129-135, 2000.

ZHU, Q.; SARKIS, J. Relationships between operational practices and performance among early adopters of green supply chain management practices in Chinese manufacturing enterprises. Journal of Operations Management, v. 22, p. 265-289, 2004. ZHU, Q.; SARKIS, J.; GENG, Y. Green supply chain management in China: pressures, practices and performance. International Journal of Operations and Production Management, v. 25, n. 5, p. 449-68, 2006.

WANG, Y. F.; CHEN, S. P.; LEE, Y. C.; TSAI, C. T. Developing green management standards for restaurants: An application of green supply chain management.

International Journal of Hospitality Management, v.34, p. 263-273, 2013. 